\title{
ON A GENERALIZATION OF AN INEQUALITY OF HARDY, LITTLEWOOD, AND PÓLYA ${ }^{1}$
}

\author{
MILTON SOBEL
}

Two sets of $n$ real numbers each are given:

$$
A=\left\{a_{1} \leqq a_{2} \leqq \cdots \leqq a_{n}\right\}, \quad B=\left\{b_{1} \leqq b_{2} \leqq \cdots \leqq b_{n}\right\} .
$$

These numbers will be regarded as distinct although two or more may be equal in value. Let $n_{i}(i=1,2, \cdots, k)$ be any fixed set of $k$ positive integers whose sum is $n$, let $C=\prod_{i=1}^{k} n_{i}$ ! and let $R=n ! / C$. We shall consider partitions $P^{*}=\left\{B_{1}^{*}, B_{2}{ }^{*}, \cdots, B_{k}^{*}\right\}$ of the set $B$ into disjoint subsets $B_{i}^{*}$, each $B_{i}^{*}$ containing $n_{i}$ elements of $B$ $(i=1,2, \cdots, k)$. By a partition here is meant an ordered $k$-tuplet of subsets, i.e. we consider two partitions $P^{*}, P^{* *}$ to be the same (or equal) if and only if $B_{i}^{*}=B_{i}^{* *}(i=1,2, \cdots, k)$. The number of such partitions is given by the multinomial coefficient $R$. If we let $B_{1}^{1}$ dedenote the first $n_{1}$ elements of $B, B_{2}^{1}$ the next $n_{2}$ elements of $B, \cdots$, $B_{k}^{1}$ the last $n_{k}$ elements of $B$, then $P^{1}=\left\{B_{1}^{1}, B_{2}^{1}, \cdots, B_{k}^{1}\right\}$ is a particular partition of $B$. Similarly if we let $B_{1}^{R}$ denote the last $n_{1}$ elements of $B, B_{2}^{R}$ the next $n_{2}$ elements of $B, \cdots, B_{k}^{R}$ the first $n_{k}$ elements of $B$, then $P^{R}=\left\{B_{1}^{R}, B_{2}^{R}, \cdots, B_{k}^{R}\right\}$ is another particular partition of $B$. Let $A_{i}^{1}=A_{i}(i=1,2, \cdots, k)$ be defined similarly for the set $A$, except that we shall regard the $A_{i}$ as ordered subsets of $A$, the order being that given in (1). For convenience we define $N=\{1,2, \cdots, n\}$ and the subsets $N_{i}^{1}=N_{i}(i=1,2, \cdots, k)$ exactly as was done for $B$.

The theorem that follows is concerned with the $n$ ! cross products $\sum_{i=1}^{n} a_{i} b_{j_{i}}$ where $\left(j_{1}, j_{2}, \cdots, j_{n}\right)$ is a rearrangement of $(1,2, \cdots, n)$. Corresponding to any fixed partition $P^{r}(r=1,2, \cdots, R)$ we consider the set $V_{r}=\left\{v_{c r} \mid c=1,2, \cdots, C\right\}$ of the $C$ cross products obtained by associating the elements of $B_{i}^{r}$ with $A_{i}(i=1,2, \cdots, k)$, all possible rearrangements within the subsets $B_{i}^{r}$ being allowed. The $n$ ! cross products are thus divided in $R$ sets with $C$ cross products in each set.

Let $P^{r}(r=1,2, \cdots, R)$ denote an arbitrary enumeration of the $R$ partitions except that $P^{1}$ and $P^{R}$ are defined above. We shall introduce (see The partial ordering below) a partial ordering (written $\left.P^{*}>P^{* *}\right)$ among the partitions such that

Presented to the Society, October 24, 1953; received by the editors November 23, 1953 and, in revised form, January 29, 1954.

1 This research was sponsored by the United States Air Force under Contract No. AF 18(600)331 monitored by the Office of Scientific.Research. 


$$
P^{1}>P^{r}>P^{R} \quad(r=2,3, \cdots, R-1) .
$$

Theorem 1. There exists a two-way table with $R$ rows and $C$ columns such that, for any pair $\left(r_{1}, r_{2}\right)$, if $P^{r_{1}}>P^{r_{2}}$ then

$$
v_{c r_{1}} \geqq v_{c r_{2}} \quad(c=1,2, \cdots, C) .
$$

Corollary 1. For each $r(r=1,2, \cdots, R)$ there exists a one-to-one pairing of the $v_{c r}$ with the $v_{c 1}$ and also with the $v_{c R}$ such that

$$
v_{c 1} \geqq v_{c r} \geqq v_{c R} \quad(c=1,2, \cdots, C) .
$$

Proof. This follows immediately from (2) and Theorem 1, the columns of the two-way table providing the required one-to-one pairing.

Corollary 2. For the special case $n_{i}=1(i=1,2, \cdots, k)$ and hence $n=k$ we have for all rearrangements $\left(j_{1}, j_{2}, \cdots, j_{n}\right)$ of $(1,2, \cdots, n)$

$$
\sum_{i=1}^{n} a_{i} b_{i} \geqq \sum_{i=1}^{n} a_{i} b_{j_{i}} \geqq \sum_{i=1}^{n} a_{i} b_{n-i+1} .
$$

Proof. If $n_{i}=1(i=1,2, \cdots, k)$ then $C=1, R=n !$ and the result follows from Corollary 1 . This is a known result given in $[1$, Theorem 368 .

The partial ordering. Consider any partition $P^{r}$. We shall denote by a left exchange the operation of interchanging the positions in $P^{r}$ of two elements $b_{p} \in B_{s}$ and $b_{q} \in B_{t}$ with say $p<q$ and having the following properties:

1. The two elements belong to different subsets in $P^{r}$, i.e. $s \neq t$.

2. The $b$ 's are going out of their natural order (1), i.e. $s<t$, and $p<q$.

Then define $P^{r_{1}}>P^{r_{2}}$ if there exists a nonempty ordered set (or product) of left exchanges that will take $P^{r_{1}}$ into $P^{r_{2}}$. The properties of reflexiveness, transitivity, and antisymmetry are easily shown and hence the partitions form a partially ordered system (p.o.s.).

Some interesting properties of the p.o.s. thus formed were noted. For all values of $n, k$ and $n_{1}, n_{2}, \cdots, n_{k}$ the p.o.s. thus formed appears to satisfy the constant chain condition, i.e. that all chains connecting the same 2 elements have the same length. For $k=2$ the p.o.s. forms a lattice while for $k>2$ it does not. These properties have not been proved (they are not used here) and therefore should be regarded as conjectures based on observed results. We also note that in all cases the replacement of every $b_{i}$ by $b_{n-i+1}$ reverses all inequalities and explains the symmetry that is always present.

We shall define a unit left exchange to be a left exchange of $b_{p} \in B_{s}$ 
and $b_{q} \in B_{t}$ such that none of the subsets $B_{s}, B_{s+1}, \cdots, B_{t}$ contains an element $b_{x}$ with $p<x<q$. It will be seen below that this defines an immediate successor in the above p.o.s. If we define right exchange and unit right exchange similarly except that the $b$ 's go into their natural order (1), then a product of right exchanges and a unit right exchange correspond respectively to predecessor and immediate predecessor in the above p.o.s. We shall later have occasion to consider the subsets $B_{i}(i=1,2, \cdots, k)$ as ordered. Then, clearly, a property of the unit exchanges is that they leave the ordering in each $B_{i}$ unaltered.

To see that a unit left exchange corresponds to an immediate successor, consider any non-unit left exchange which takes $P^{\prime}$ into $P^{\prime \prime}$. Suppose the elements exchanged are $b_{p} \in B_{s}$ and $b_{q} \in B_{t}$. Then there is an element $b_{x}$ in one of the sets $B_{s}, B_{s+1}, \cdots, B_{t}$ with $p<x$ $<q$. If $b_{x} \notin B_{s}$, then define $P^{*}$ as the partition $P^{\prime}$ with $b_{x}$ and $b_{q}$ interchanged; if $b_{x} \in B_{s}$, then define $P^{*}$ as the partition $P^{\prime}$ with $b_{x}$ and $b_{p}$ interchanged. In either case we then have

$$
P^{\prime}>P^{*}>P^{\prime \prime} \text {. }
$$

Conversely if the exchange connecting two partitions $P^{\prime}$ and $P^{\prime \prime}$ is a unit exchange then it is simple but tedious to show that there exists no partition $P^{*}$ satisfying (6). We omit this proof.

Proof of Theorem 1. To prove (2) we shall first show that any given partition $P^{r}$ can be reached by starting with $P^{1}$ and performing a sequence of left exchanges. It then follows from the previous paragraph that $P^{r}$ can be reached by starting with $P^{1}$ and performing a sequence of $u n i t$ left exchanges. Label each element $b_{j}(j=1,2, \cdots, n)$ in $P^{1}$ as a left-mover, right-mover, or non-mover according to the direction it has to move in order to change $P^{1}$ to $P^{r}$. Let $b_{y}$ be the first left-mover reading from left-to-right. Then there must be at least one right-mover to the left of $b_{y}$ which is not in the same subset as $b_{y}$; let $b_{z}$ be that particular one of these which is farthest to the right. Exchange $b_{y}$ and $b_{z}$ and then relabel all the $b_{j}$ again as left-, right-, or non-movers. Repeat the procedure until $P^{r}$ is formed. By this procedure left-movers (right-movers) continue moving to the left (right) until they become non-movers. It follows that each step of the procedure satisfies properties 1 and 2 and is therefore a left exchange. This proves the first part of (2); the second part follows similarly if we start with $P^{R}$ and proceed by means of right exchanges to $P^{r}$.

Each of the $n$ ! cross products $\sum_{i=1}^{n} a_{i} b_{j_{i}}$ can be uniquely identified by the associated permutation 


$$
\left(\begin{array}{ll}
1, & 2, \cdots, n \\
j_{1}, & j_{2}, \cdots, j_{n}
\end{array}\right)
$$

and the set of all these forms the symmetric group $S_{n}=S(N)$ on the $n$ elements of $N$. Let $S\left(N_{i}\right)$ denote the symmetric group on the $n_{i}$ elements of $N_{i}(i=1,2, \cdots, k)$. Form the subgroup $G$ of $S_{n}$

$$
G=S\left(N_{k}\right) \times S\left(N_{k-1}\right) \times \cdots \times S\left(N_{1}\right)
$$

which is the direct product of these $k$ symmetric groups. It should be noted that the elements of $G$ correspond to the $C$ cross products $v_{c 1}$ of $V_{1}(c=1,2, \cdots, C)$.

We shall divide the group $S_{n}$ into $R$ cosets (corresponding to the rows of our table) modulo the subgroup $G$. Since $G$ consists of all the permutations within the subsets $B_{1}^{1}, B_{2}^{1}, \cdots, B_{k}^{1}$, it is clear that each partition $P^{r}$ will correspond to a row, say the $r$ th row, of our table. The first row of our table will then consist of the cross products corresponding to the elements $g_{c 1}$ of $G$ in any order except that, for convenience, we set $g_{11}$ equal to the identity. The order within each of the remaining rows will then be determined by an induction on the p.o.s.

Suppose that the order of the cross products in the $r_{1}$ th row, corresponding to $P^{r_{1}}$, has been determined; let $g_{c r_{1}}$ denote the corresponding permutations. Suppose also that $P^{r_{2}}$ is an immediate successor of $P^{r_{1}}$ in the p.o.s. and that the associated unit exchange involves $b_{p}$ and $b_{q}$ with $p<q$. Then define the permutation

$$
g_{c r_{2}}=(p q) g_{c r_{1}} \quad(c=1,2, \cdots, C)
$$

as the product of $g_{c r_{1}} b y$ the transposition $(p q)$. This determines the order of the cross product entries in the $r_{2}$ th row, corresponding to $P^{r_{2}}$.

We first show that, for any pair $\left(r_{1}, r_{2}\right)$, if $P^{r_{1}}>P^{r_{2}}$ then there exists a one-to-one pairing of $\left\{v_{r_{1}}\right\}$ and $\left\{v_{c r_{2}}\right\}$ such that

$$
v_{c r_{1}} \geqq v_{c r_{2}} \quad(c=1,2, \cdots, C) .
$$

It suffices to assume that $P^{r_{2}}$ is an immediate successor of $P^{r_{1}}$ in the p.o.s. Let $b_{p} \in B_{s}$ and $b_{q} \in B_{t}$ in $P^{r_{1}}$ be the elements of $B$ exchanged. Assume $p<q$. Then, by (1), $b_{p} \leqq b_{q}$ and by property 2 we have $s<t$. Hence for any fixed $c$ it follows that the coefficients of $b_{p}$ and $b_{q}$ in $v_{c r_{1}}$, say $a_{c p}$ and $a_{c q}$, belong to the distinct subsets $A_{s}$ and $A_{t}$, respectively. Hence $a_{c p} \leqq a_{c q}$. It is easy to see that for each $c$ the above exchange affects only terms involving $b_{p}$ and $b_{q}$ and in fact that

$$
v_{c r_{1}}-v_{c r_{2}}=\left(a_{c q}-a_{c p}\right)\left(b_{q}-b_{p}\right) \geqq 0 \quad(c=1,2, \cdots, C) .
$$


This proves (9).

To complete the proof of the theorem we need only consider partitions of $P^{r_{2}}$ which have more than one immediate predecessor, say $P^{r_{1}}$ and $P^{r_{0}}$, in the p.o.s. and show that the above ordering of the $r_{2}$ th row is the same regardless of which predecessor is used. By (2) we can refer these two methods to the common starting point $P^{1}$ and write for one method

$$
\stackrel{1}{g_{c r_{2}}}=g_{1} g_{c 1} \quad(c=1,2, \cdots),
$$

and for the other

$$
g_{c r_{2}}^{0}=g_{0} g_{c 1} \quad(c=1,2, \cdots, C) .
$$

It follows from (11) and (12) that $g_{0}$ and $g_{1}$ are in the same coset. The problem is to show that $g_{0}=g_{1}$. If $g_{11}$ is the identity it is sufficient by (11) and (12) to show that $g_{1 r_{2}}^{1}=g_{1 r_{2}}^{0}$.

$\mathrm{Up}$ to this point the order within the subsets $B_{i}^{*}$ in $P^{*}$ $=\left\{B_{1}^{*}, B_{2}^{*}, \cdots, B_{\mathbb{k}}^{*}\right\}$ has not been considered but by taking this order into account we can identify the individual cross products in each row and hence also the corresponding permutations. For example if the partition with ordering within subsets is $\left\{\left(b_{1}\right) ;\left(b_{3}, b_{2}\right)\right\}$, then the corresponding cross product is $a_{1} b_{1}+a_{2} b_{3}+a_{3} b_{2}$ and the corresponding permutation is

$$
\left(\begin{array}{lll}
1 & 2 & 3 \\
1 & 3 & 2
\end{array}\right)=(23)
$$

Let us start at the top of our p.o.s. with $P^{1}=\left\{B_{1}^{1}, B_{2}^{1}, \cdots, B_{k}^{1}\right\}$, each subset $B_{\imath}^{1}$ being in natural order (1), and consider the effects of the various exchanges as we travel down different "paths" leading to $P^{r_{2}}$. It was noted above that each unit exchange leaves the order within the subsets unaltered. Then $g_{1 r_{2}}^{1}$ and $g_{1 r_{2}}^{0}$ correspond to the same partition with the same ordering within subsets and hence they are equal. This completes the proof of the theorem.

Corollary 3. If in addition to (1) we have no two b's equal and

$$
a_{i}<a_{i+1} \quad\left(i=n_{1}, n_{1}+n_{2}, \cdots, \sum_{i=1}^{k} n_{i}\right),
$$

then Theorem 1 holds with strict inequality for each $c(c=1,2, \cdots, C)$.

Proof. Under the added hypothesis strict inequality holds for both factors in (10) and the result follows.

This problem arose in connection with a statistical problem [2] 
involving a sequential multiple decision procedure for ranking parameters. Two corollaries of particular interest in [2] will now be given.

Corollary 4. If $P^{r_{1}}>P^{r_{2}}$, then for each $c(c=1,2, \cdots, C)$ we can write $v_{c r_{1}}-v_{c r_{2}}$ as a sum of non-negative terms in the form

$$
v_{c r_{1}}-v_{c r_{2}}=\sum\left(b_{q}-b_{p}\right)\left(a_{w}-a_{u}\right)
$$

where

$$
q \geqq w>u \geqq p .
$$

Proof. Label each element $b_{j}(j=1,2, \cdots, k)$ in $P^{r_{1}}$ as a left-, right- or non-mover according to its position in $P^{r_{2}}$. Proceed as in the proof of (2) above to find a sequence of left exchanges leading from $P^{r_{1}}$ to $P^{r_{2}}$. To each exchange in turn we can apply the same argument as was used to obtain (10). To prove (15) we refer to the property of this method, noted in the proof of (2), that left-movers (rightmovers) continue to move to the left (right). Since $b_{q}$ is a left-mover and $a_{w}$ is its "temporary position" it follows that $q \geqq w$. Similarly, $b_{p}$ being a right-mover, $p \leqq u$. By properties 1 and 2 we have that $a_{u}$ and $a_{w}$ belong to different subsets, say $a_{u} \in A_{s}$ and $a_{w} \in A_{t}$, and that $s<t$. It follows that $u<w$.

For the last corollary we consider the special case $k=2$ which is the case that arises in the application [2] and define $b_{0}=-\infty$ and $b_{n+1}=+\infty$. We shall assume in addition to (1) that

$$
\begin{aligned}
b_{n_{1}-m_{1}}<b_{n_{1}-m_{1}+1} & =b_{n_{1}-m_{1}+2}=\cdots=b_{n_{1}}=b_{n_{1}+1}=\cdots \\
& =b_{n_{1}+m_{2}}<b_{n_{1}+m_{2}+1}
\end{aligned}
$$

where $1 \leqq m_{1} \leqq n_{1}$ and $0 \leqq m_{2} \leqq n_{2}$.

Corollary 5. (a) If $m_{1}$ and $m_{2}$ are defined by (16), then the two-way table of Theorem 1 will contain $C_{m_{2}}^{m_{1}+m_{2}}$ rows, including the first row, such that entries in the same column are equal. (b) If $m_{1}+m_{2}<n$ and

(i) $a_{n_{1}}<a_{n_{1}+1}$,

(ii) not all the a's are equal,

then comparing any one of the remaining rows with the first row we will have in cases (i) and (ii) respectively

(i) strict inequality in each column,

(ii) strict inequality in at least one column.

Proof. (a) By Theorem 1, the first and largest row is obtained by associating with the fixed set $A_{2}=\left\{a_{n_{1}+1}, a_{n_{1}+2}, \cdots, a_{n}\right\}$ the set or combination $B_{2}^{1}=\left\{b_{n_{1}+1}, b_{n_{1}+2}, \cdots, b_{n}\right\}$ as coefficients. Clearly, ex- 
changing any $b$ in $B_{2}^{1}$ with another $b$ equal in value from $B_{1}^{1}$ will not affect any of the cross products $v_{c 1}(c=1,2, \cdots, C)$. Hence by (16) there are $C_{m_{2}}^{m_{1}+m_{2}}$ rows, including the first row, that are equivalent.

(b) If $m_{1}+m_{2}<n$, then not all the b's are equal and there is at least one more row in our two-way table not included in (a), i.e. there is at least one more partition $P^{r_{0}}$ not included in (a). We can assume, for simplicity, that the partition $P^{r_{0}}$ differs from $P^{1}$ by an exchange of $b_{p}$ and $b_{q}$ where either $p \leqq n_{1}-m_{1}$ and $q>n_{1}$ or $p \leqq n_{1}$ and $q>n_{1}+m_{2}$. This assumption is permitted since any partition $P^{r_{0}}$ not in (a) differs from $P^{1}$ by a product of exchanges, at least one of which is of the above type. Then $b_{p}<b_{q}$. For each $c(c=1,2, \cdots, C)$ we exchange $b_{p}$ and $b_{q}$ in $v_{c 1}$ to form $v_{c r_{0}}$. If we let $a_{c p}$ and $a_{c q}$ denote the coefficients of $b_{p}$ and $b_{q}$ respectively in $v_{c 1}$, then clearly, for case (i), $a_{c q}>a_{c p}$ for every $c$ and, for case (ii), $a_{c q} \geqq a_{c p}$ for every $c$ with strict inequality holding for at least one $c$. Then we obtain as in (10) above

$$
v_{c 1}-v_{c r_{0}}=\left(a_{c q}-a_{c p}\right)\left(b_{q}-b_{p}\right) \geqq 0 \quad(c=1,2, \cdots, C) .
$$

For case (i) we have strict inequality for each $c$ and for case (ii) we have strict inequality for at least one $c$.

The ordering of row $r_{0}$ described above must be the same as the ordering in Theorem 1 since the left exchange of $b_{p}$ with $b_{q}$ can be expressed as a product of unit left exchanges.

Acknowledgment. The author wishes to thank Mr. Walter Feit and Dr. J. K. Goldhaber of Cornell University for helpful discussions on the material of this paper.

\section{REFERENCES}

1. G. H. Hardy, J. E. Littlewood, and G. Pólya, Inequalities, London, Cambridge University Press, 1934.

2. R. E. Bechhofer, M. Sobel, and J. C. Kiefer, On a sequential multiple decision procedure for certain identification and ranking problems (to be submitted to Ann. Math. Statist.).

Cornell University 